\title{
Une nouvelle formule pour notre revue
}

\section{(2) OpenEdition}

1 Journals

Édition électronique

URL : http://journals.openedition.org/anthropodev/783

DOI : 10.4000/anthropodev.783

ISSN : 2553-1719

Éditeur

Presses universitaires de Louvain

\section{Édition imprimée}

Date de publication : 1 décembre 2019

Pagination : 7-8

ISBN : 978-2-87558-940-8

ISSN : 2276-2019

Référence électronique

"Une nouvelle formule pour notre revue », Anthropologie \& développement [En ligne], 50 | 2019, mis en ligne le 16 juin 2020, consulté le 25 janvier 2021. URL : http://journals.openedition.org/anthropodev/ 783 ; DOI : https://doi.org/10.4000/anthropodev.783

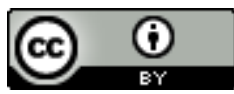

La revue Anthropologie \& développement est mise à disposition selon les termes de la Licence Creative Commons Attribution 4.0 International. 


\section{Une nouvelle formule pour notre revue}

Le Bureau de l'APAD et le Comité de rédaction

Ce numéro 50 marque une nouvelle étape dans la vie de notre revue. Fondé en même temps que I'APAD, en 1991, le Bulletin de l'APAD a connu plusieurs formules avant de devenir, en 2013, Anthropologie \& développement. Achevant la trans-formation, déjà engagée les années précédentes, du Bulletin en revue bilingue à comité de lecture, cette nouvelle formule était auto-éditée par l'association. Six ans après, la revue étant stabilisée, I'APAD a souhaité faire un pas de plus dans sa professionnalisation. Anthropologie \& développement est désormais publiée par les Presses universitaires de Louvain (PUL). L'impression et la diffusion sont assurées par le Centre d'impression et $\mathrm{d}^{\prime}$ achat en coopérative ( $\left.\mathrm{ClACO}\right)$, prestataire des PUL. Le choix de s'associer à des presses universitaires ainsi qu'à une coopérative s'inscrit bien dans les valeurs portées par l'APAD.

Cette évolution s'accompagne d'un changement dans le format et la périodicité de la revue. Grâce à la compétence des PUL et de CIACO, et au passage de la reprographie à l'imprimerie, le format a été agrandi, la maquette rénovée, la lisibilité améliorée, la qualité du papier accrue. Pensée à l'origine comme une revue semestrielle, alternant les numéros thématiques et varia, la revue a surtout produit des numéros doubles. Nous avons choisi de publier désormais un seul volume par an, de la taille des anciens numéros doubles, combinant un dossier et des articles en varia ${ }^{1}$. Nous espérons que vous apprécierez cette nouvelle formule, qui devrait faciliter la publication des articles hors dossier.

L'association avec CIACO facilite la diffusion de notre revue, qui était auparavant uniquement disponible à la vente sur le site de l'APAD. C'était une des motivations pour faire évoluer notre formule. Désormais, l'achat en ligne est aisé sur le site des PUL ou sur i6doc.com, qui diffuse une trentaine de revues scientifiques. Tout libraire peut commander un exemplaire, via les réseaux de diffusion classiques du livre.

\footnotetext{
${ }^{1}$ Pour des raisons liées au temps de production des numéros, cette formule ne sera pleinement effective qu'à partir de 2021, avec le numéro " Le capitalisme, les entrepreneurs et les associations d'entrepreneurs au travail : nouvelles ethnographies des dynamiques du secteur privé en Afrique ", coordonné par Thomas Bierschenk et José María Muñoz (voir : https://apad-association.org/le-capitalisme-les-entrepreneurs-etles-associations-dentrepreneurs-au-travail-nouvelles-ethnographies-des-dynamiques-du-secteur-prive-enafrique/).
} 
Enfin, jusqu'alors essentiellement portée par l'implication bénévole d'un petit nombre de membres du bureau de l'association, l'organisation interne de la rédaction évolue. La composition du comité de rédaction s'élargit. Barbara Bentz, Gabriella Körling et Fatoumata Ouattara ont passé le relais après des années d'engagement soutenu. Nous ne saurons trop les remercier pour leur engagement sans lequel la revue n'aurait pas tenu depuis 2013. Alexis Roy a pris la suite en tant que rédacteur en chef et a engagé un élargissement du comité. Nous souhaitons la bienvenue à Sophie Andreetta, Thomas Bierschenk, Nehara Feldman, Pauline Jarroux, Cristiano Lanzano, Jacinthe Mazzocchetti, Emmanuelle Piccoli et Jessica Pourraz. Enfin, face au travail que représente la vie d'une revue, Marie-Christine Polge, qui réalise depuis 2014 le travail d'editing des articles et de mise en page de la revue, assure désormais en plus le secrétariat de rédaction.

Le présent numéro est constitué d'articles varia. La variété des sujets abordés illustre l'ampleur et la richesse des thèmes de préoccupation de la revue: la question de la résilience, abordée par le cas des jeunes filles maliennes en situation de migration urbaine (Bouju) ; l'action publique autour des inondations dans les quartiers populaires de Dakar (Leclercq); les dynamiques d'associations musulmanes de migrants nigérians en Côte d'Ivoire (Binaté et Dao) ; une réflexion sur la catégorie " enfants des rues " et ses effets sur leur prise en charge au Sénégal (Diack) ; la socialisation sexuelle des jeunes issus des migrations subsahariennes en Suisse (Villani, Poglia Mileti, Mellini et Sulstarova); une expérience de microfinance en Tanzanie (Baroin).

Il comprend aussi les textes des conférences plénières présentées au dernier colloque de l'APAD, organisé à l'université de Roskilde en mai 2018 sur les enjeux migratoires. Engin Isin questionne le biais des représentations autour de la sédentarité, montre l'importance des mobilités, et discute l'intérêt de penser la catégorie des " gens mobiles » comme un concept politique. Étudiant les questions d'asile en Grèce depuis une dizaine d'années, bien avant la " crise » de 2015, Heath Cabot propose de s'intéresser aux vagues et aux reflux des humanitaires et des chercheurs travaillant sur les migrations et les réfugiés, et la façon dont certains moments sont qualifiés de "crise ", justifiant un afflux des uns et des autres. Ces deux textes, indisponibles jusqu'ici en français, débutent la valorisation scientifique du colloque, qui se poursuivra dans le prochain numéro.

Enfin, le prochain grand événement organisé par l'APAD sera son prochain colloque, qui se déroulera à Lomé, du 23 au 26 juin 2020. L'APAD propose pour cette édition, coorganisée avec le département d'anthropologie et d'études africaines de la faculté des sciences de l'homme et de la société (FSHS) de l'université de Lomé (Togo), de centrer ses travaux sur les multiples formes de circulation à grande échelle de marchandises, idées, techniques, informations, modèles qui traversent de manière croissante les pays du Sud et les relient au reste du monde ${ }^{2}$. Nous espérons vous y retrouver nombreux!

\footnotetext{
${ }^{2}$ https://apad-association.org/colloques/colloque-2020/
} 